\title{
Theory of Synthetic Aperture Radar Imaging of a Moving Target
}

\author{
Jen King Jao, Member, IEEE
}

\begin{abstract}
Two novel image processing techniques have been developed to refocus a moving target image from its smeared response in the synthetic aperture radar (SAR) image which is focused on the stationary ground. Both approaches may be implemented with efficient fast Fourier transform (FFT) routines to process the Fourier spatial spectrum of the image data. The first approach utilizes a matched target filter that is derived from the signal history along the range-Doppler migration path mapped onto the SAR image from the moving target trajectory in real space. The coherent spatial filter is specified by the apparent target range in the image and the magnitude of the relative target-to-radar velocity. The second approach eliminates the range-dependence by reconstructing the moving target image from a spectral function that is obtained from the SAR image data spectrum via a spatial frequency coordinate transformation.
\end{abstract}

\section{INTRODUCTION}

A IRBORNE radar detection of moving targets on the ground has been a subject of long-standing interest. Moving target detection is usually accomplished by enhancing the contrast between the target Doppler signature and the competing ground clutter returns via Doppler filtering or by applying the displaced phase center antenna (DPCA) and its modern extension, the space-time adaptive processing (STAP) techniques [1]-[3] for clutter cancellation. While these clutter suppression techniques are generally effective in improving the detectability of a fast target, the detection of a slow target remains a very challenging endeavor. As the target radial velocity along the radar line-of-sight (LOS) slows down and the target Doppler diminishes, the target signal will eventually be masked by the strong "mainbeam" ground clutter spectrum. Although either STAP or DPCA technique, supported by a multichannel receiver system and a receiving antenna of multiple phase centers, may in principle cancel both sidelobe and mainbeam clutter, nevertheless, it is difficult to achieve high clutter cancellation close to the "DC" clutter at zero Doppler without suppressing at the same time the target signal as well. Because the clutter bandwidth is roughly proportional to both the radar platform speed and the radar antenna beamwidth, the detection of a slower ground mover at a small minimum discernible velocity (MDV) fundamentally requires a long antenna or a narrow azimuthal beamwidth, a requirement

\footnotetext{
Manuscript received August 3, 2000; revised May 1, 2001. This work was supported in part by the Defense Advanced Research Project Agency (DARPA) under U.S. Air Force Contract F19628-95-C-0002 under a program sponsored by DARPA at the Massachusetts Institute of Technology's Lincoln Laboratory, and the Army/CECOM, Soler, Inc.

The author is with the Lincoln Laboratory, Massachusetts Institute of Technology, Lexington, MA 02420-9185 USA.

Publisher Item Identifier S 0196-2892(01)07619-7.
}

that is sometimes impractical to fulfill. The alternative is to extend the traditional synthetic aperture radar (SAR) approach for moving target detection and imaging. The airborne SAR employs a very different signal processing paradigm to improve the target-to-clutter contrast. The SAR integrates in a long coherent pulse interval (CPI) over a large signal bandwidth and synthesizes a long synthetic aperture along the radar flight track in order to form a high-resolution SAR image of the ground without the need of a long antenna in the azimuth dimension. Thus, radar target signal gain is enhanced while the strength of the ground backscatter is reduced through the reduction of the ground clutter cell area. The increasing radar sensitivity is gained at the expense of longer coherent dwell time or, eventually, slower area coverage rate. One shortcoming of the conventional SAR approach, from the perspective of the moving target detection, lies in its image processing that, by design, focuses on a stationary target scene [4]-[6]; a moving target appears in the image unfocused or smeared with a considerable loss of the target-to-clutter ratio.

Recent advances in the ultrawideband (UWB) SAR, particularly for the foliage and ground penetration applications, has demonstrated both the required radar technology and processing techniques necessary to support the imaging resolution on the order of one radar wavelength $\lambda$ or better [7]-[16]. In a typical situation, a large number of broad-band pulses are transmitted and the signals backscattered from a ground target are gathered over a long radar flight time interval or distance that may span a large azimuth angle $\theta$ looking from the radar toward the intended target. The matched filtering operation, such as pulse compression, over the large signal bandwidth $B$ results in a high range resolution, on the order of $c / 2 B, c$ being the speed of light. Whereas a high cross range resolution, on the order of $\lambda / 2 \theta$, is realized by integrating all the target pulse returns collected within the radar look angle $\theta$ corresponding to the synthetic radar aperture $D$. However, because of the long synthetic radar aperture length, or correspondingly, the large integration angle and the long CPI, the process of cross range data compression or target focusing requires precise compensation of the aircraft motion, the target range-Doppler migration, and the phase curvature due to the spherical propagation path of the target echoes [4]-[6]. Henceforth, an SAR processing scheme that is designed to form a high-resolution image of a stationary target must necessarily be matched to the signal phase history of the intended target as observed over the radar CPI. Accordingly, the same processing scheme and parameters used to image a stationary target will naturally be mismatched to that required to process the moving target returns. As the kinematics of a moving target relative to the SAR differ from that of a stationary target, 
so will their respective signal phase histories over the radar CPI. This dissimilarity results in the smearing of the moving target signature in the SAR image focused on a stationary scene.

In an SAR stationary ground image, a moving target appears to be relocated in cross range due to the radial range velocity and defocused due to both the along-track velocity and the radial range acceleration [17]. Several SAR moving target imaging approaches have been proposed in previous investigations [18]-[25]. These approaches generally attempt to estimate the motion parameters from the SAR target data and then, based on the estimated parameters and a signal model, compensate the quadratic and higher order target phase observed in the video phase history data in order to focus a moving target. Unfortunately, it is often difficult to accurately estimate the parameters of an unfocused target due to insufficient contrast between the target and the strong clutter background. It has been suggested that better target parameter estimation accuracy may be achieved by forming a sequence of subaperture images as a moving target is expected to be only moderately defocused in a subaperture image. However, the validity of this proposition remains to be proven in practice. Of course, optimal target detection and parameter estimation, as two aspects of the same matched filtering process, are not truly separable. In principle, the moving target imaging, filtering, detection, and estimation may be formulated as a process to test multiple hypotheses of various target motion parameters. Realistically, this formulation is impractical since the number of possible motion hypotheses may be too large. Even for a simple rectillinear motion, a unique target state must be specified by both its initial position and the velocity vectors; thus, without any prior knowledge of the target state, the parameter search must be conducted in a four-dimensional space.

The following sections present the theory of SAR imaging for moving target focusing. Immediately following a short exposition of the SAR imaging formation via backprojection, the inverse filtering process of moving target focusing will be explained in three key conceptual steps that are graphically illustrated by three panels in Fig. 1. The first step is to develop a model to predict the target migration path that is essentially a mapping of the moving target trajectory in real space onto the SAR image domain as shown by the image data example in Fig. 1(a). The second step, illustrated in Fig. 1(b), is to define the image phase structure along the target migration path; the target phase response is subsequently used to define a moving target image filter. The third step explains two different spectral domain implementations of refocusing a moving target with one resulting example shown in Fig. 1(c).In this paper, a new approach of moving target imaging is proposed and analyzed. As opposed to previous investigations, this new approach reconstructs the moving target signature by processing the SAR image instead of video data. Treating the SAR image as the output of a filter, one may regard the image response to a moving target as the convolution of the target signal and the SAR point spread function matched to the signal of a stationary point object on the ground. While the initial SAR processing may not take into account the target movement and, as a consequence, results in a blurred target image, nonetheless, the image is expected to retain all pertinent information that characterizes the target motion his-
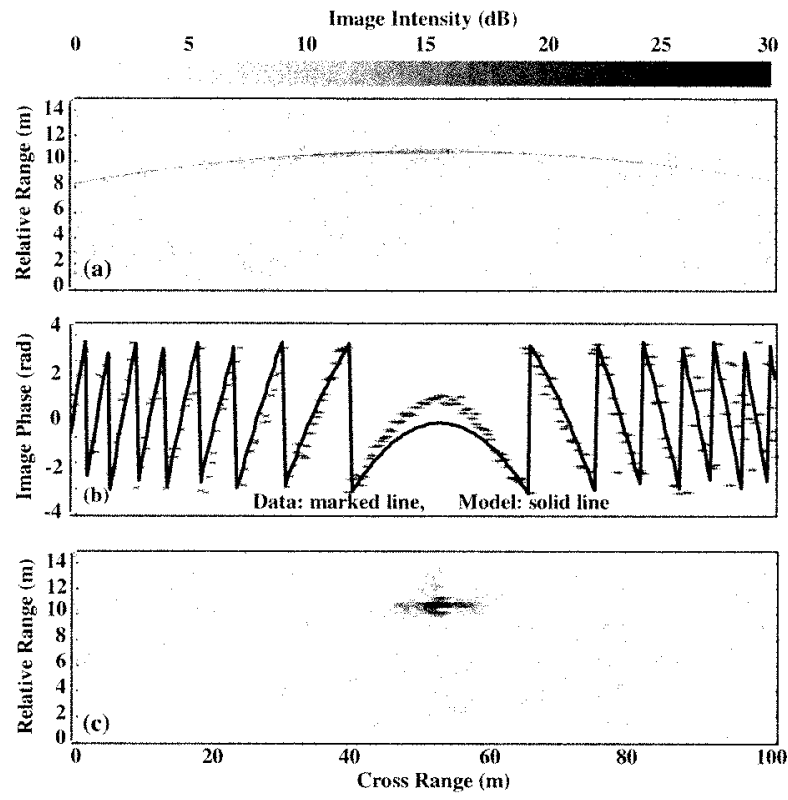

Fig. 1. Example of the P3 SAR image.

tory. Therefore, by understanding the target image response and its parametric dependence on the motion parameters, it should be possible to reconstruct the target signal via proper deconvolution. Indeed, it will be shown in this paper that the target migration path can be predicted from the target motion parameters. The phase history along the migration path in the SAR image will be used to define a matched filter to refocus the moving target image. One surprising result emerging from the following analysis is that, other than the apparent target range in the image, the matched filter is uniquely specified by the magnitude of the relative target-to-radar velocity or speed. Further, the matched filter can be extended into a fast Fourier transform (FFT)-based processing scheme that eliminates the parametric dependence on the target range. This spectral processing scheme focuses the target image via the inverse Fourier transform of a spectral function that is created by interpolating the SAR image data spectrum in a new coordinate grid [26]-[28]. Consequently, the parameter search space required for moving target processing becomes one-dimensional (1-D). The filter bank required in practice to focus a prospective moving target needs to cover only the expected range of the relative target-to-radar speed.

The SAR image in Fig. 1(a) is focused on the stationary ground. The image, plotted in the cross range and the slant range coordinate plane, is processed from the US Navy/P-3 UWB radar data gathered over a bandwidth of $515 \mathrm{MHz}$ from 215 to $730 \mathrm{MHz}$. The SAR integration angle is close to $30^{\circ}$ [14] and given the image slant range of more than $5000 \mathrm{~m}$ and the aircraft speed of about $130 \mathrm{~m} / \mathrm{s}$, the coherent integration time is approximately $20 \mathrm{~s}$, The image range and cross range pixel spacing, respectively sampled at 0.235 and $0.4 \mathrm{~m}$, is finer than the nominal SAR image resolution (of $0.33 \times 0.66 \mathrm{~m}$ ). Clearly, adequate aircraft motion compensation is critical and must be properly applied to radar data processing so as to obtain the diffraction-limited image resolution comparable to the radar center wavelength of about $0.6 \mathrm{~m}$. Fortunately, motion compensation with sufficient accuracy over a long radar flight 


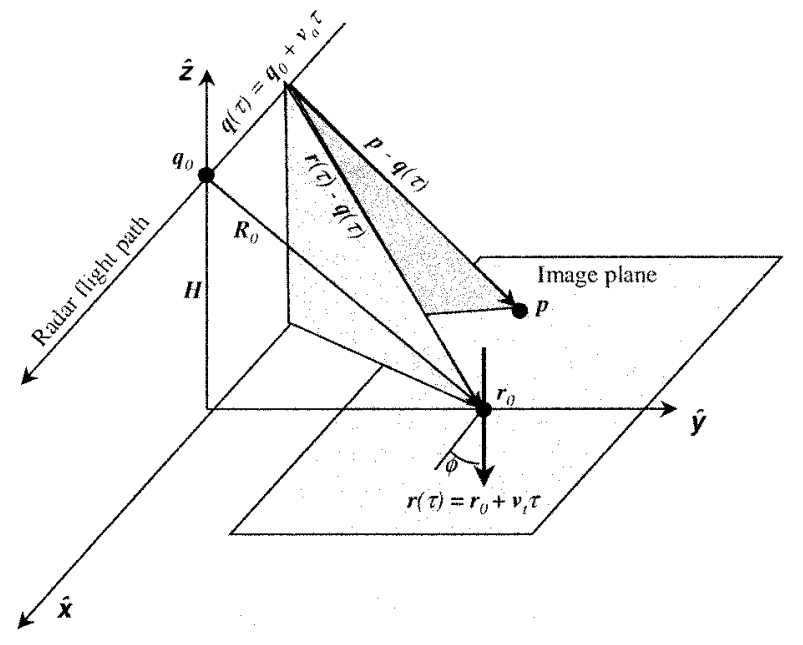

Fig. 2. SAR imaging geometry.

path or integration time is practically feasible as the ultrahigh frequency SAR is more tolerant of motion-induced errors. The depicted image of $64 \times 256$ pixels shows the image response to a moving truck-sized vehicle close to the top center with its broadside nearly facing the radar. The vehicle moves at the approximate speed of $7 \mathrm{~m} / \mathrm{s}$ toward the opposite radar heading direction. As expected, the unfocused signature of the moving truck, smeared over a curvilinear path in the image, bears little resemblance to that of a small target. Thus, the image processing task at hand is to reconstruct the unfocused moving target signature into a focused target image.

\section{SAR IMAGE FORMATION}

In this section, the SAR imaging process is analyzed for the strip-map geometry shown in Fig. 2. However, it is worthwhile to point out that the ensuing analysis and results are equally applicable to the spotlight SAR imaging geometry.

In Fig. 2, the ground plane is defined by two Cartesian unit vectors, $\hat{\mathbf{x}}$ and $\hat{\mathbf{y}}$, and its surface normal by $\hat{\mathbf{z}}$. The radar aircraft's flight path, following the heading along $\hat{\mathrm{X}}$, is represented by the position vector $\mathbf{q}(\tau)$. The variable $\tau$ is the "slow time" that marks the time of radar pulse emission within the SAR flight time interval $\left[T_{0}, T_{1}\right]$. In addition, the notations $\nu_{0}, \lambda_{0}=$ $c / \nu_{0}$, and $k_{0}=2 \pi / \lambda_{0}=2 \pi \nu_{0} / c$ will be used to denote the radar center frequency and the corresponding wavelength and wavenumber. In order to simplify the definition of the radar signal phase history between $\left[T_{0}, T_{1}\right]$, the radar position $\mathbf{q}(\tau)$ will be assumed to be frozen during each time instant $\tau$ of pulse transmission and reception. Under this circumstance, the radar pulse with an instantaneous bandwidth $B$ about $\nu_{0}$ is transmitted, backscattered from a single ground point scatterer at $\mathbf{r}$, received, and processed by a typical chain of RF downconversion, IF bandpass filtering, range compression via matched filtering, video quadrature demodulation, and digital sampling above the Nyquist rate $1 / B$ at multiple "fast time" instants $t$. This process yields a complex signal sample $g(t, \tau, \mathbf{r})$ as a function of $\mathbf{r}, \tau$, and $t$ corresponding to the slant range $c t / 2$. Using a range spread function $p_{r}$ to represent the output of the range compression filter with a range resolution on the order of $c / 2 B$, one may write the sample signal $g$ as

$$
\begin{gathered}
g(t, \tau, \mathbf{r})=\frac{b(\tau, \mathbf{r})}{R^{2}(\tau, \mathbf{r})} \exp \left[j 2 \pi \nu_{0}\left(t-\frac{2}{c} R(\tau, \mathbf{r})\right)\right] \\
\times p_{r}\left(t-\frac{2}{c} R(\tau, \mathbf{r})\right) \\
R(\tau, \mathbf{r})=|\mathbf{r}-\mathbf{q}(\tau)| .
\end{gathered}
$$

Here, the slowly varying $R(\tau, \mathrm{r})$ stands for the slant range of the radar LOS from $\mathbf{q}(\tau)$ to $\mathbf{r}$. The amplitude variable $b(\tau, \mathbf{r})$ lumps together all the effects, other than spherical propagation, that may affect the signal amplitude due to variation in the target backscatter, antenna gain, etc. Although in a realistic situation $b(\tau, \mathbf{r})$ may fluctuate as the relative radar and target positions change in time $\tau$, nevertheless, $b(\tau, \mathbf{r})$ will be taken as a nonfluctuating and nondispersive constant $b$ throughout this paper as this assumption will not affect the validity of the following target imaging analysis.

In the SAR image formation process, the response $f(\mathbf{p})$ at an image point $\mathbf{p}=\left(p_{x}, p_{y}\right)$ is obtained by integrating all the radar signal samples $g(t, \tau, \mathbf{r})$ over $\tau \in\left[T_{0}, T_{1}\right]$ after proper compensation of the time-delay $(2 / c)|\mathbf{p}-\mathbf{q}(\tau)|$. For a pulsed radar, the integration sums up a discrete number of signal samples, but for reason of clarity, an equivalent, continuous backprojection formulation [6] will be adopted. In this treatment, the SAR image point spread function $f(\mathbf{p})$, normalized to have a maximum unity amplitude, may be written as

$$
\begin{aligned}
f(\mathbf{p})= & C \int_{T_{0}}^{T_{1}} g\left(\frac{2}{c}|\mathbf{p}-\mathbf{q}(\tau)|, \tau, \mathbf{r}\right) d \tau \\
f(\mathbf{p})= & C \int_{T_{0}}^{T_{1}} \frac{b(\tau, \mathbf{r})}{R^{2}(\tau, \mathbf{r})} \\
& \times \exp \left(j 2 k_{0}(|\mathbf{p}-\mathbf{q}(\tau)|-|\mathbf{r}-\mathbf{q}(\tau)|)\right) \\
& \times p_{r}\left(\frac{2}{c}(|\mathbf{p}-\mathbf{q}(\tau)|-|\mathbf{r}-\mathbf{q}(\tau)|)\right) d \tau \\
& C=\left|\int_{T_{0}}^{T_{1}} \frac{b(\tau, \mathbf{r})}{R^{2}(\tau, \mathbf{r})} d \tau\right|^{-1} .
\end{aligned}
$$

It is obvious from this equation that, given a single stationary point scatterer at $\mathbf{r}$, the peak response of $f(\mathbf{p})$ occurs at the location $\mathbf{p}=\mathbf{r}$ that coincides with that of the scatterer. However, the response of $f(\mathrm{p})$ to a moving scatterer requires more careful deliberation.

\section{Moving TARgEt IMAgE RESPONSE}

Consider now the case of the radar and a ground target moving at the respectively constant but different velocity, $\mathbf{v}_{a}$ and $\mathbf{v}_{t}$, along two different trajectories $\mathbf{q}(\tau)$ and $\mathbf{r}(\tau)$

$$
\begin{aligned}
\mathbf{q}(\tau) & =\mathbf{q}_{0}+\mathbf{v}_{a} \tau \\
\mathbf{r}(\tau) & =\mathbf{r}_{0}+\mathbf{v}_{t} \tau . \\
\mathbf{q}_{0} & =H \hat{\mathbf{z}} \\
\mathbf{r}_{0} & =r_{0} \hat{\mathbf{y}} .
\end{aligned}
$$


Without losing generality, the coordinate frame as shown in Fig. 2 will be assumed to have been suitably arranged such that the coordinates of two reference points, $\mathbf{q}_{0}$ for the aircraft flight path $\mathbf{q}(\tau)$ and $\mathbf{r}_{0}$ for the target trajectory $\mathbf{r}(\tau)$, will both lie in the same $(\mathbf{y}, \mathbf{z})$ plane. These vectors along with the relative target-to-radar slant range vectors, $\mathbf{R}(\tau)$ and $\mathbf{R}_{0}$, and the target velocity relative to the radar $\mathbf{v}$ are explicitly defined as

$$
\begin{aligned}
\mathbf{R}(\tau) & =\mathbf{r}(\tau)-\mathbf{q}(\tau)=\mathbf{R}_{0}+\mathbf{v} \tau \\
\mathbf{R}_{0} & =\mathbf{r}_{0}-\mathbf{q}_{0}=r_{0} \hat{\mathbf{y}}-H \hat{\mathbf{z}} \\
\mathbf{v} & =\mathbf{v}_{t}-\mathbf{v}_{a} .
\end{aligned}
$$

Substituting $\mathrm{r}$ in (2) with $\mathbf{r}(\tau)$, the SAR image response $f(\mathbf{p})$ to a moving point target now becomes

$$
\begin{aligned}
f(\mathbf{p})= & C \int_{T_{0}}^{T_{1}} \frac{b(\tau, \mathbf{r})}{R^{2}(\tau, \mathbf{r})} \\
& \times \exp \left(j 2 k_{0}(|\mathbf{p}-\mathbf{q}(\tau)|-|\mathbf{r}(\tau)-\mathbf{q}(\tau)|)\right) \\
& \times p_{r}\left(\frac{2}{c}(|\mathbf{p}-\mathbf{q}(\tau)|-|\mathbf{r}(\tau)-\mathbf{q}(\tau)|)\right) d \tau
\end{aligned}
$$

Because of the time varying target position $\mathbf{r}(\tau)$, (5) will not produce a focused moving target image at a single image point $\mathbf{p}=\mathbf{r}_{0}$ unless the aircraft motion relative to the target be properly compensated by replacing $|\mathbf{p}-\mathbf{q}(\tau)|$ with $\left|\mathbf{p}-\mathbf{q}(\tau)+\mathbf{v}_{t} \tau\right|$. In fact, the above imaging response projects the rectilinear target trajectory $\mathbf{r}(\tau)$ in the real space onto a curvilinear curve in the SAR image.

\section{Moving TARget Migration}

The curvilinear migration path in the SAR image can be determined as the sequence of the image points $\mathbf{p}_{t}$ that maximizes the response $f(\mathbf{p})$ of (5) to the target backscatter moving along the trajectory $\mathbf{r}(\tau)$. Intuitively, one may regard $\mathbf{p}_{t}$ as the focal point of the subaperture target image integrated over a very small but finite time interval $\left[T_{a}, T_{b}\right] \subseteq\left[T_{0}, T_{1}\right]$ containing the time instant $\tau$. The trace of $\mathbf{p}_{t}$ is generated by progressively sliding $\left[T_{a}, T_{b}\right]$ over the entire SAR aperture $\left[T_{0}, T_{1}\right]$. The local maximum of $f(\mathbf{p})$ occurs at the point $\mathbf{p}=\mathbf{p}_{t}$ under two conditions: 1) the phase of the exponential term in (5) becomes stationary, and 2) the argument of the range spread function $p_{r}$ vanishes.

The first stationary phase condition [29, p. 76], valid under the circumstance that the slant range of the image area is much larger than the wavelength $\lambda$, requires the following derivative to vanish at $\mathbf{p}_{t}$ :

$$
\left.\left[\frac{d}{d \tau}(|\mathbf{p}-\mathbf{q}(\tau)|-|\mathbf{r}(\tau)-\mathbf{q}(\tau)|)\right)\right]_{\mathbf{p}=\mathbf{p}_{t}}=0
$$

or explicitly

$$
\frac{-\dot{\mathbf{q}}(\tau) \cdot\left[\mathbf{p}_{t}-\mathbf{q}(\tau)\right]}{\left|\mathbf{p}_{t}-\mathbf{q}(\tau)\right|}=\frac{[\dot{\mathbf{r}}(\tau)-\dot{\mathbf{q}}(\tau)] \cdot[\mathbf{r}(\tau)-\mathbf{q}(\tau)]}{|\mathbf{r}(\tau)-\mathbf{q}(\tau)|}
$$

By multiplying both sides of (6) with $2 / \lambda$, it is clear that this expression equates the target Doppler (on the right side) observed by the radar to the Doppler shift of the ground point $\mathbf{p}_{t}$ (on the left side). At the time instant $\tau$, the target position will be translated to one point on the ground Doppler isodop corresponding to the target Doppler shift. Similarly, the second condition

$$
\left|\mathbf{p}_{t}-\mathbf{q}(\tau)\right|=|\mathbf{r}(\tau)-\mathbf{q}(\tau)| .
$$

projects the target to a ground point $\mathbf{p}_{t}$ on the circular iso-range contour relative to the radar. Therefore, the intersection of the Doppler isodop and iso-range contour, determined from the correct target Doppler and range, will map the target position $\mathbf{r}(\tau)$ onto a unique image point $\mathbf{p}_{t}$.

With some algebraic manipulations and using the notations defined in (4)-(7) and the parameter $\alpha$

$$
\alpha=1-\frac{v_{a}^{2}}{v^{2}}, \quad v_{a}=\left|\mathbf{v}_{a}\right|, \quad v=|\mathbf{v}|
$$

the target migration path is parametrically obtained as

$$
\begin{aligned}
& p_{t x}=-r_{0} \frac{v_{y}}{v_{a}}-\alpha \frac{v^{2} \tau}{v_{a}} \\
& p_{t s}^{2}=R_{0}^{2}-p_{t x}^{2}-\alpha v^{2} \tau^{2}, \quad R_{0}=\sqrt{r_{0}^{2}+H^{2}}
\end{aligned}
$$

in terms of the cross range $p_{t x}=\mathbf{p}_{t} \cdot \hat{\mathbf{x}}$, the ground range $p_{t y}=$ $\mathbf{p}_{t} \cdot \hat{\mathbf{y}}$, and the slant range $p_{t s}=\sqrt{p_{t y}^{2}+H^{2}}$ of the image point $\mathbf{p}_{t}$, as well as the ground range component of the relative target velocity $v_{y}=\mathbf{v} \cdot \hat{\mathbf{y}}$. By eliminating the slow-time parameter $\tau$, the above expressions can be converted into a quadratic equation that specifies the moving target migration trajectory in the SAR image as

$$
\begin{aligned}
& p_{t s}=\left[R_{s}^{2}-\frac{1}{\alpha}\left(p_{t x}-R_{c}\right)^{2}\right]^{1 / 2} \\
& R_{s}=\left[R_{0}^{2}-\left(r_{0} \frac{v_{y}}{v}\right)^{2}\right]^{1 / 2}=\left[H^{2}+r_{0}^{2}\left(1-\frac{v_{y}^{2}}{v^{2}}\right)\right]^{1 / 2} \\
& R_{c}=-r_{0} \frac{v_{a} v_{y}}{v^{2}} .
\end{aligned}
$$

Note from (10) that the apparent slant range $R_{s}$ of the target or the apex of the conic migration curve in the image is less than the true target range $R_{0}$; the discrepancy varies with $\left|r_{0} v_{y} / v\right|$. A similar relation also holds between the apparent and the true ground range $r_{0}$ of the target. In addition, there is an apparent cross range displacement $R_{c}$ equal to $-r_{0} v_{a} v_{y} / v^{2}$. Because of the Doppler shift due to $-v_{y}$, the cross range of the reference point $R_{c}$ moves either forward or backward in the image for, respectively, an approaching $\left(v_{y}<0\right)$ or departing $\left(v_{y}>0\right)$ target.

The moving target trajectory is either elliptical for a positive $\alpha$ or hyperbolic for a negative $\alpha$; the sign of $\alpha$ depends on the ratio of the relative target speed $v$ and the radar aircraft speed $v_{a}$. For an in-bound target approaching the radar, $v$ is larger than $v_{a}$ and $\alpha$ is positive. Consequently, the target migration path is a convex downward ellipse as the curve illustrated by 


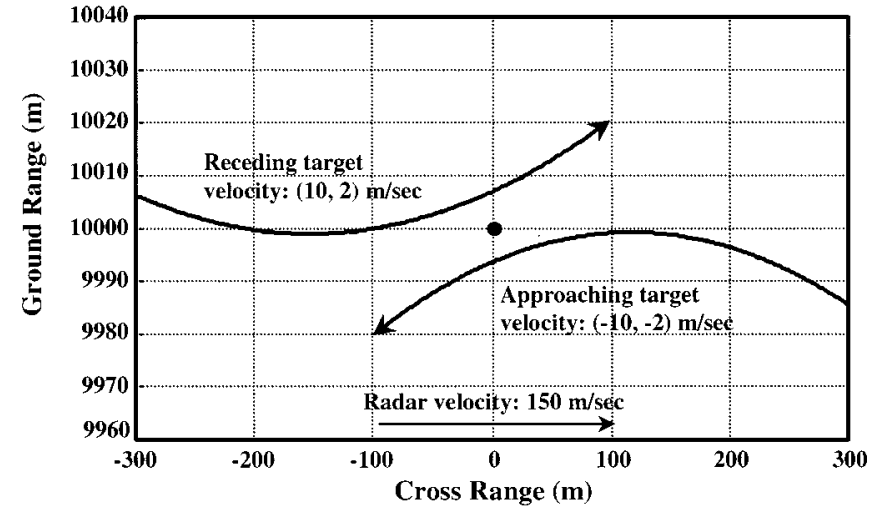

Fig. 3. Target migration paths in the SAR image. The solid curve is for an approaching and the dashed curve is for an advancing target.

Fig. 1(a). Conversely, for an out-bound target departing from the radar, $v$ is less than $v_{a}$ and $\alpha$ becomes negative. In this case the target migration curve is a concave upward hyperbola. These two situations are graphically illustrated by Fig. 3. In this figure, two target migration plots are shown in the cross range and the ground range coordinate plane, $\left(p_{t x}\right.$ and $\left.p_{t y}\right)$. The assumed radar speed is $150 \mathrm{~m} / \mathrm{s}$ moving in the direction of $\hat{\mathbf{x}}$, and the target reference ground range is $r_{0}=10 \hat{y} \mathrm{~km}$. Two cases are depicted: the target velocity for the first case is $\mathbf{v}_{t}=-10 \hat{\mathbf{x}}-2 \hat{\mathbf{y}}$ $\mathrm{m} / \mathrm{s}$, and the second is $\mathbf{v}_{t}=10 \hat{\mathbf{x}}+2 \hat{\mathbf{y}} \mathrm{m} / \mathrm{s}$.

\section{TARget Image Signal AND SPECTRUM}

Along the target trajectory $\mathbf{p}_{t}$, the complex image signal amplitude $f\left(\mathbf{p}_{t}\right)$ is equal to unity under the equal Doppler and range constraints of (6) and (7). This is obtained from (5) by ignoring the scattering amplitude fluctuation $b(\tau, \mathrm{r})$ over $\left(T_{0}, T_{1}\right)$ and the slowly varying range dependence $1 / R^{2}(\tau, \mathbf{r})$ of the target signal. However, $f\left(\mathrm{p}_{t}\right)$ may generally be modeled as

$$
f\left(\mathbf{p}_{t}\right)=\exp \left(j 2 \gamma k_{0} p_{t s}\right) ; \quad \gamma=0, \pm 1 .
$$

Here, the choice of the $\gamma$ value is implementation-specific but needs to be consistent with the SAR mechanization and image focusing scheme. The presence or absence of the range-dependent "carrier phase" factor $2 k_{0} p_{s}$ depends on whether this factor in the target video phase history is retained in the SAR cross range focusing operation and the sign of $\gamma$ reflects the relative phase of SAR video quadrature signals. For the P-3 radar data example illustrated in Fig. 1(b), $2 k_{0} p_{t s}$ is used to represent the image phase data. It is evident from this figure that the predicted phase progression along the target migration path, plotted as the solid curve, corroborates the experimental data, plotted as the marked curve.

By knowing the moving target migration trajectory and its signal phase structure along the path, it becomes possible to refocus the blurred target image and to reconstruct its signature. The process involves the construction of a moving target signal template followed by its convolution with the SAR image data. For this purpose, consider again the same equation (10) in a new reference frame by translating the origin of the image cross range and slant range coordinates $\mathbf{p}=\left(p_{x}, p_{s}\right)$ to $\mathbf{p}_{0}=$
$\left(R_{c}, R_{s}\right)$. Using an overbar to represent a vector in this new coordinate frame

$$
\begin{aligned}
\overline{\mathbf{p}} & =\mathbf{p}-\mathbf{p}_{0}, \\
\overline{\mathbf{p}}_{t} & =\mathbf{p}_{t}-\mathbf{p}_{0}, \quad \mathbf{p}_{0}=\left(R_{c}, R_{s}\right)
\end{aligned}
$$

the moving target image vector $\overline{\mathbf{p}}_{t}$ is now governed by a simplified functional relationship $\bar{p}_{t s}=T\left(\bar{p}_{t x} ; R_{s}, v\right)$ between the differential slant range $\bar{p}_{t s}$ and cross range $\bar{p}_{t x}$ coordinates

$$
\bar{p}_{t s}=T\left(\bar{p}_{t x} ; R_{s}, v\right)=\left[R_{s}^{2}-\frac{\bar{p}_{t x}^{2}}{\alpha}\right]^{1 / 2}-R_{s} .
$$

From the above migration trajectory, the moving target image impulse response $h\left(\overline{\mathbf{p}} ; R_{s}, v\right)$ and its Fourier spectrum $H\left(\mathbf{k} ; R_{s}, v\right)$ can be represented by

$$
\begin{aligned}
h\left(\overline{\mathbf{p}} ; R_{s}, v\right)= & \delta\left(\overline{\mathbf{p}}-\overline{\mathbf{p}}_{t}\right) \exp \left(j 2 \gamma k_{0} p_{t s}\right) \\
\beta_{s}= & k_{s}-2 \gamma k_{0} \\
H\left(\mathrm{k} ; R_{s}, v\right)= & \int_{-\infty}^{\infty} \int_{-\infty}^{\infty} h\left(\overline{\mathbf{p}} ; R_{s}, v\right) \exp (-j \mathrm{k} \cdot \overline{\mathbf{p}}) d \overline{\mathbf{p}} \\
= & \exp \left(j 2 \gamma k_{0} R_{s}\right) \\
& \times \int_{-\infty}^{\infty} \sqrt{1+T^{2}\left(\bar{p}_{t x} ; R_{s}, v\right)} \\
& \times \exp \left(-j k_{x} \bar{p}_{t x}-j \beta_{s} T\left(\bar{p}_{t x} ; R_{s}, v\right)\right) d \bar{p}_{t x} .
\end{aligned}
$$

Here, $\mathbf{k}=\left(k_{x}, k_{s}\right)$ denotes the image cross range and slant range angular frequency vector. The derivative $T^{\prime}\left(\bar{p}_{t x} ; R_{s}, v\right)$ with respect to $\bar{p}_{t x}$ is the slope of the target migration curve. Applying the method of stationary phase $[29$, p. 76] to the above equation, $H\left(\mathrm{k} ; R_{s}, v\right)$ can be shown to be

$$
\begin{aligned}
H\left(\mathbf{k} ; R_{s}, v\right)= & C(\mathbf{k}) \\
& \times \exp \left(j k_{s} R_{s}-j \operatorname{sign}\left(\beta_{s}\right) \sqrt{\alpha k_{x}^{2}+\beta_{s}^{2}} R_{s}\right) \\
C(\mathbf{k})= & \frac{\left(2 \pi|\alpha|\left(k_{x}^{2}+\beta_{s}^{2}\right)\right)^{1 / 2} R_{s}}{\left(\alpha k_{x}^{2}+\beta_{s}^{2}\right)^{3 / 4}} \exp \left(j \frac{\pi}{4} \operatorname{sign}\left(\alpha \beta_{s}\right)\right) .
\end{aligned}
$$

The exponential factor in the above expression specifies the phase structure of the target spectrum that is the key to define the moving target focusing filter. Note that both $h\left(\overline{\mathbf{p}} ; R_{s}, v\right)$ and $H\left(\mathbf{k} ; R_{s}, v\right)$ are functions of $R_{s}$ and $v$.

\section{MOVING TARGET FoCUSING}

Knowing the SAR image response of a moving target, the moving target signature can now be focused onto the image position $\mathbf{p}_{0}=\left(R_{c}, R_{s}\right)$ as $f_{t}\left(\mathbf{p}_{0}\right)$ by applying a range-variant filter. This task is accomplished by carrying out the spatial convolution of the target impulse response with the SAR image data 


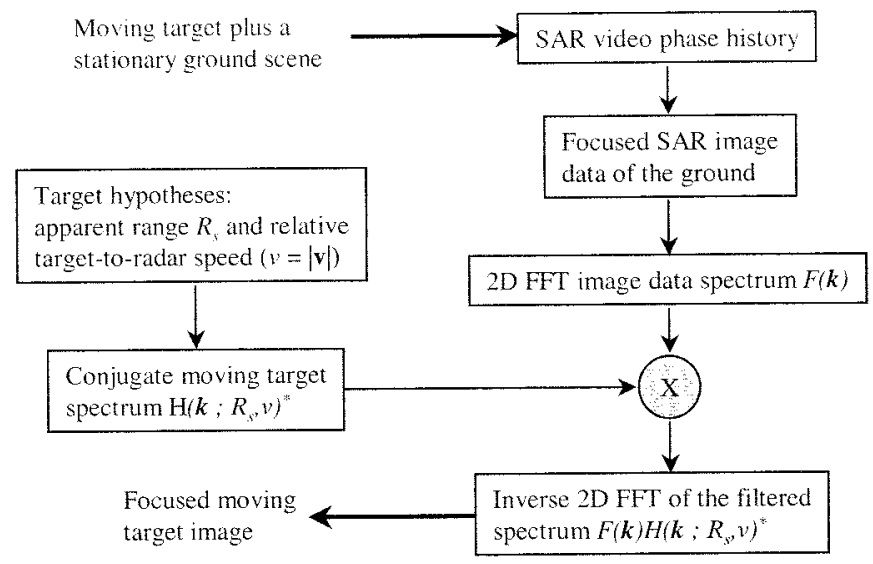

Fig. 4. SAR image filtering for moving target refocusing.

$f(\mathbf{p})$ or, alternatively, by performing the inverse Fourier transform of the SAR image data spectrum $F(\mathbf{k})$ filtered by the conjugated target signal spectrum $H^{*}\left(\mathbf{k} ; R_{s}, v\right)$

$$
\begin{aligned}
f_{t}\left(\mathbf{p}_{0}\right)= & \int_{-\infty}^{\infty} \int_{-\infty}^{\infty} f(\mathbf{p}) h^{*}\left(\mathbf{p}-\mathbf{p}_{0} ; R_{s}, v\right) d \mathbf{p} \\
= & \frac{1}{(2 \pi)^{2}} \int_{-\infty}^{\infty} \int_{-\infty}^{\infty} F(\mathbf{k}) H^{*}\left(\mathbf{k} ; R_{s}, v\right) \\
& \times \exp \left(j \mathbf{k} \cdot \mathbf{p}_{0}\right) d \mathbf{k} .
\end{aligned}
$$

Clearly, the filtering process can be efficiently implemented with the FFT operations as illustrated by the block diagram in Fig. 4. Using this processing paradigm, the result of one moving target imaging example is shown in Fig. 1(c). This panel depicts the spatial-filtered output by applying the matched target spectrum of (15) to the input SAR image data. Notice that the moving target has been refocused from its smeared counterpart in Fig. 1(a).

Because of the range dependence of the focusing filter, in principle, one must replace $R_{s}$ in $H\left(\mathrm{k} ; R_{s}, v\right)$ by an appropriate range parameter in order to focus a prospective target located at a different position. However, it turns out that, similar to the range migration or the $\omega k$ technique previously developed for the SAR image formation [26]-[28], a range-invariant procedure may be formulated to eliminate the range-dependence of the above process. In this approach, the focused target image is obtained as the inverse Fourier transform of the image data spectrum $F(\mathbf{k})$, provided the spectrum is first projected onto a different spatial frequency coordinate system. This process requires spectral interpolation or a coordinate transformation similar to the Stolt transformation employed by the $\omega k$ technique.

In this context, one may compute $f_{t}\left(\mathbf{p}_{0}\right)$ as the inverse Fourier transform of the spectral function $F_{t}(\mathbf{K})$ defined in term of a new spatial frequency vector $\mathbf{K}=\left(K_{x}, K_{r}\right)$

$$
f_{t}\left(\mathbf{p}_{0}\right)=\frac{1}{(2 \pi)^{2}} \int_{-\infty}^{\infty} \int_{-\infty}^{\infty} F_{t}(\mathbf{K}) \exp \left(j \mathbf{K} \cdot \mathbf{p}_{0}\right) d \mathbf{K}
$$

By ignoring the slowly varing dependence of $C(\mathbf{k})$ on the range variable $R_{s}, F_{t}(\mathbf{K})$ may be approximately obtained from (15)

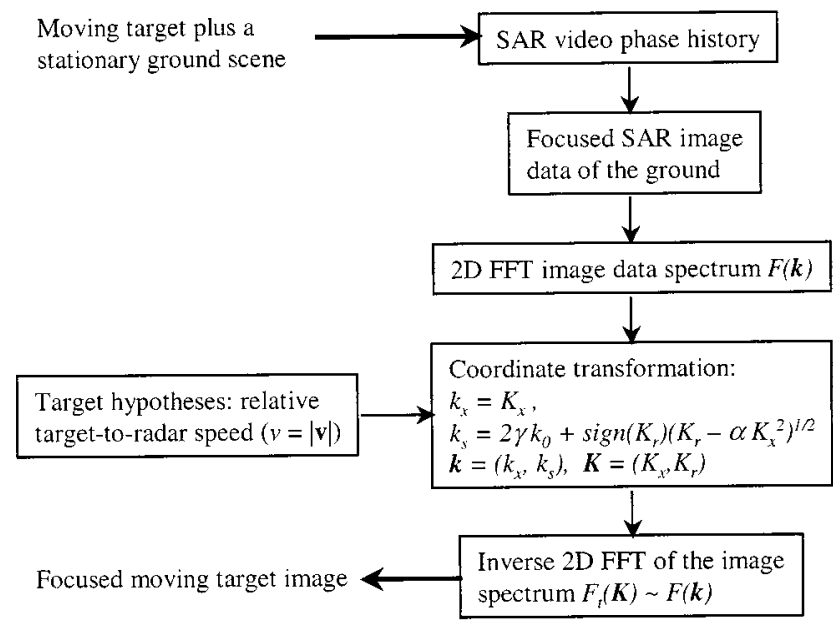

Fig. 5. Moving target focusing via image spectral data processing.

and (16) as

$$
\begin{aligned}
F_{t}(\mathbf{K})= & \frac{1}{(2 \pi)^{2}} \int_{-\infty}^{\infty} \int_{-\infty}^{\infty} \int_{-\infty}^{\infty} \int_{-\infty}^{\infty} F(\mathbf{k}) \\
& \times H^{*}\left(\mathbf{k} ; R_{s}, v\right) \exp \left(j(\mathbf{k}-\mathbf{K}) \cdot \mathbf{p}_{0}\right) d \mathbf{p}_{0} d \mathbf{k} \\
\simeq & \int_{-\infty}^{\infty} \int_{-\infty}^{\infty} C^{*}(\mathbf{k}) F(\mathbf{k}) \delta\left(K_{x}-k_{x}\right) \\
& \times \delta\left(K_{r}-\operatorname{sign}\left(\beta_{s}\right) \sqrt{\alpha k_{x}^{2}+\beta_{s}^{2}}\right) d \mathbf{k} .
\end{aligned}
$$

Thus, $F_{t}(\mathbf{K})$ can be calculated by interpolating the image data spectrum $F(\mathbf{k})$ according to the following coordinate transformation between $\mathbf{k}=\left(k_{x}, k_{s}\right)$ and $\mathbf{K}=\left(K_{x}, K_{r}\right)$

$$
\begin{aligned}
F_{t}(\mathbf{K}) & =\frac{\sqrt{\alpha k_{x}^{2}+\beta_{s}^{2}}}{\left|\beta_{s}\right|} C^{*}(\mathbf{k}) F(\mathbf{k}), \quad \text { for } \quad K_{x}=k_{x}, \\
K_{r} & =\operatorname{sign}\left(\beta_{s}\right) \sqrt{\alpha k_{x}^{2}+\beta_{s}^{2}} \text { or } \\
k_{s} & =2 \gamma k_{0}+\operatorname{sign}\left(K_{r}\right) \sqrt{K_{r}^{2}-\alpha K_{x}^{2}} .
\end{aligned}
$$

The FFT implementation of the above range-invariant spectral processing scheme from (17)-(19) is further explained in Fig. 5.

As a practical matter, one often needs to apply a phase correction to both the data and the image spectra should the popular FFT be used in the spectral computation. The data spectrum $F(\mathbf{k})$ is actually the product of the exponential factor $\exp \left(-j k_{s} R_{\text {min }}\right)$ and the FFT of a finite SAR image patch $f(\mathrm{p})$ that covers a range swath starting from a minimum slant range $R_{\text {min }}$ larger than zero. Similarly, the resulting image spectrum $F_{t}(\mathbf{K})$ must be multiplied by the factor $\exp \left(j K_{r} R_{\min }\right)$ before the inverse FFT to generate the moving target image. Including these corrections and using the same coordinate transformation for $k_{s}$ and $K_{r},(19)$ is now replaced by

$$
\begin{aligned}
F_{t}\left(K_{x}, K_{r}\right)= & \frac{\sqrt{\alpha k_{x}^{2}+\beta_{s}^{2}}}{\left|\beta_{s}\right|} C^{*}\left(K_{x}, k_{s}\right) \\
& \times \exp \left(-j k_{s} R_{\min }+j K_{r} R_{\min }\right) F\left(K_{x}, k_{s}\right) .
\end{aligned}
$$


It is interesting to note that, in the new spectral coordinates $\left(K_{x}, K_{r}\right)$, the moving target image spectrum is the product of the transformed SAR image data spectrum and a filter transfer function; the latter has a spectral response similar to (15).

\section{DISCUSSION}

In the preceding section, a coherent spatial filter has been defined that may be used to process the SAR image of a stationary scene to refocus the moving target image. Conventional SAR image formation is designed to focus a stationary target by sampling and compensating the quadratic and higher order target phase curvature over the synthetic aperture. The mismatch between the stationary target focusing process and the target motion defocuses and smears the moving target image response along a curvilinear trajectory, a phenomenon reminiscent of the well-known target range walk or migration in the SAR video history data prior to image focusing. However, the SAR image retains all the information about the target motion; consequently, a smeared moving target can be refocused by deconvolving the SAR image data. Indeed, given the rectilinear target movement at a constant velocity $\mathbf{v}_{t}$, the preceding analysis predicts a conic migration trajectory in the SAR image via the range-Doppler mapping. The image migration path, with its apex located at the apparent target range $R_{s}$, is either a concave-downward ellipse or convex-upward hyperbola, depending, respectively, on whether the ratio between the radar aircraft speed $v_{a}=\left|\mathbf{v}_{a}\right|$ and the relative target-to-radar speed $v=\left|\mathbf{v}_{t}-\mathbf{v}_{a}\right|$ is smaller or larger than unity. Along the migration trajectory, the moving target image signal is characterized by a modulation factor $\exp \left(2 j \gamma k_{0} p_{t s}\right), \gamma=0, \pm 1$ with a linear slant range dependent phase. This characterization faithfully captures the image phase structure of a moving object that enables the construction of a useful target filter from the image impulse response of (14) and its spectrum of (15).

The spatial filtering approach clearly has the benefit of computational efficiency due to the readily available FFT routine. Using the exact range-variant focusing filter, the pair of parameters, $R_{s}$ and $\alpha$, must be matched to those of the intended target. Otherwise, the deviation from the target truth will result in a mismatched filter that may not properly focus the target image. The required accuracy and sensitivity in selecting appropriate parameters for target focusing will be radar and target specific. It is conceivable that, in the general case, a bank of filters spanning a suitable range of $v$ must be used to process one cross range slice of the image data at a different range $R_{s}$. Also, it has been shown that the range-variant filtering may be replaced by a range-invariant processing scheme. The latter process accomplishes the moving target focusing in three sequential steps including a Fourier transform of the SAR image data, a mapping of the data spectrum onto different spatial frequency coordinates, and an inverse Fourier transform of the new interpolated image spectrum.

The fact that the moving target focusing process is parametrically dependent on only a single parameter $\alpha$ or $v$ has important implications. In many realistic scenarios, prior knowledge of $v$ may not always be available, consequently, a practical target-focusing solution is to adopt and test multiple values of $v$

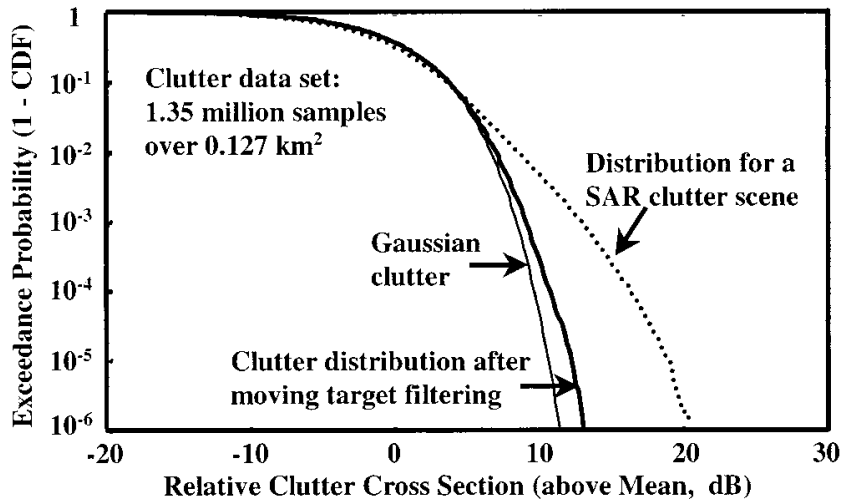

Fig. 6. Clutter distributions before and after moving target filtering.

within its expected range. If the search range of $v$ is considerably reduced so is the complexity of the spatial filter design and the resulting processing load of the filtering operations. In other words, to focus a single moving target onto its apparent image position $\left(R_{c}, R_{s}\right)$, it will be sufficient to utilize 1-D filter bank that covers the expected range of the speed variation.

The spatial filter bank may be simultaneously used for target detection and target motion parameter estimation. Out of the multiple filters parametrically indexed by $v$, the moving target image response relative to the stationary ground clutter background is maximized by the filter best matched to the correct target speed. It is also evident that the target parameter estimation via the proposed target focusing operation will be ambiguous. This is attributed to the same dependence of the target filter on the apparent range $R_{s}$ and the speed $v$ instead of the related vector counterparts $\mathbf{r}_{0}$ and $\mathbf{v}$. A reduction in the dimensionality of the target parameter search space from four to two or one also introduces the ambiguity in estimating the target vector position and velocity.

Due to the enhanced target image signal-to-clutter ratio after refocusing, moving target detection performance will be improved in the subsequent thresholding operation applied to all the filtered outputs. The filter with the output target image intensity that exceeds the detection threshold not only indicates the target presence but also provides the best estimate of the target parameters. The target detectability is expected to be enhanced for two reasons. The signal strength of a refocused target is expected to increase due to the coherent integration gain. Meanwhile, stationary targets such as discrete clutter backscatterers will be blurred at the output of a moving target filter. As a result, the moving target filter will suppress the clutter amplitude fluctuation due to strong clutter discretes and increase the target-to-clutter contrast. This desirable clutter-quenching effect is illustrated by Fig. 6 . In this figure, the clutter distribution of a tree patch is plotted in terms of the exceedance probability versus the clutter cross section above its mean level on the decibel scale. The distribution of the unfiltered SAR image intensity is plotted as the dotted curve. The data set includes about 1.35 million SAR image samples covering an area of approximately $0.127 \mathrm{~km}^{2}$. The SAR pixel spacings and resolutions are identical to those cited for Fig. 1. The filtered clutter distribution is plotted in the thick solid line. Here, the moving target filter is defined by the same parameters used to focus the moving 
target example shown in Fig. 1. For convenient comparison, the exponential intensity distribution applicable to a homogeneous clutter patch is also plotted in a thin solid line. In this clutter example, the suppression of the filtered clutter fluctuation is quite obvious.

\section{CONCLUSION}

In this paper, a novel moving target imaging technique has been developed. The new approach reconstructs the moving target signature by applying the exact spatial correlation or matched filtering solution to process the SAR image instead of its raw video data. The contribution of this paper lies in the identification of the moving target response in the SAR image domain and the corresponding spatial spectrum that lead to the explicit formulation of the image filtering processes.

The filtering process essentially refocuses a moving target from its blurred SAR image signature along a curvilinear migration path. It has been shown that, the target migration can be predicted via the range-Doppler mapping that projects the target location in the real space to its corresponding position in the SAR image. The predictable target migration trajectory in the SAR image leads to a moving target signal model that is subsequently used to formulate the target focusing filter. It is shown in the above analysis that the target spectrum captures the target motion effects through two dependent variables, the apparent target reference range $R_{s}$ and the parameter $\alpha$; given the aircraft speed $v_{a}, \alpha$ is uniquely determined by the magnitude of the relative target-to-radar velocity $v=|\mathbf{v}|$.

Thus, a moving target can be focused onto an apparent image position $\mathbf{p}_{0}=\left(R_{c}, R_{s}\right)$ by convolving the range-variant impulse response function with the SAR image data. The same result also can be obtained by performing the efficient FFT filtering of the SAR image data spectrum using a filter transfer function equal to the complex conjugate of the range-dependent target spectrum. Given a pair of parameters $R_{s}$ and $v$, both methods supply the exact moving target focusing solution. Thus, the implementation of the above focusing procedures involves the design of multiple filters based on the hypothetical values of $R_{s}$ and $v$ on a two-dimensional grid. The density or the number of the parametric hypotheses will depend on the radartarget parameters, the required accuracy, and the quality of the target image signature. Interestingly, the moving target focusing also may be accomplished by applying a range-invariant spectral process that eliminates the range dependence of the above matched filtering process. This processing technique is similar to the conventional range migration approach in the SAR image formation. The preceding analysis shows that the focused moving target image is the inverse Fourier transform of the SAR image data spectrum, provided a curvilinear transformation is first applied to the spatial frequency coordinates.

In this paper, the coherent spatial filtering scheme is demonstrated with efficient FFT implementation. The application of the proposed moving target imaging approach to limited measurement data sets has been very successful. By applying a suitable target filter in the form of (15), the smeared target in the SAR image data example is refocused with a significant improvement of the target-to-background clutter contrast.
Although this paper does not explicitly address issues related to moving target detection, estimation, and classification, it is clear that these and the imaging processes are not entirely separable. The ability to image a moving target will not only enhance the target-to-clutter ratio but also improve the performance of the former, aforementioned processes.

\section{ACKNOWLEDGMENT}

The author acknowledges helpful discussions with A. Yegulalp on the SAR backprojection image formation process. The measurement data example cited in the paper was extracted from one P-3 SAR experimental data set. Many people, including M. Toups, L. Bessette, and J. Tsay, have been involved in the preparation of the data set.

\section{REFERENCES}

[1] L. E. Brennan and I. S. Reed, "Theory of adaptive radar," IEEE Trans. Aerosp. Electron. Syst., vol. AES-9, pp. 237-252, Mar. 1973.

[2] R. Klemm, Space-Time Adaptive Processing, Principle and Applications. London, U.K.: Institution of Electrical Engineers, 1998.

[3] J. Ward, "Space-time adaptive processing for airborne radar," Tech. Rep. 1015, Mass. Inst. Technol. Lincoln Lab., (AD-A293-032-9-XAB), Lexington, MA, Dec. 13, 1994.

[4] G. Franceschetti and R. Lanari, Synthetic Aperture Radar Processing. Boca Raton, FL: CRC, 1999.

[5] W. G. Carrara, R. S. Goodman, and R. M. Majewski, Spotlight Synthetic Aperture Radar Signal Processing Algorithms. Norwood, MA: Artech House, 1995

[6] A. F. Yegulalp, "Fast backprojection algorithm for synthetic aperture radar," in Proc. IEEE Radar Conf., Apr. 1999, pp. 60-64.

[7] J. G. Fleischman, S. Ayasli, E. M. Adams, and D. R. Gosselin, "Foliage penetration experiment-Part I: Foliage attenuation and backscatter analysis of SAR imagery," IEEE Trans. Aerosp. Electron. Syst., vol. 32, pp. 135-144, Jan. 1996.

[8] M. E. Toups, S. Ayasli, and J. G. Fleschman, "Foliage penetration experiment-Part II: Analysis of foliage-induced synthetic pattern distortion," IEEE Trans. Aerosp. Electron. Syst., vol. 32, pp. 145-155, Jan. 1996.

[9] J. G. Fleischman, M. A. Worris, S. Ayasli, E. M. Adams, and D. R. Gosselin, "Foliage penetration experiment-Part III: Multichannel whitening of SAR imagery," IEEE Trans. Aerosp. Electron. Syst., vol. 32, pp. 156-164, Jan. 1996.

[10] H. Hellsten, P. Frolind, A. Gustavsson, T. Johsson, B. Larson, G. Stenstrom, B. Binder, M. I. Mirkin, and S. Ayasli, "Ultrawideband VHF SAR: Design and measurements," in Proc. SPIE Conf. Aerial Surveillance Sensing Including Obscured and Underground Object Detection, Orlando, FL, Apr. 4-6, 1994, pp. 16-25.

[11] H. Hellsten, L. M. H. Ulander, A. Gustavsson, and B. Larson, "Development of VHF CARABAS II SAR," in Proc. SPIE Conf. Radar Sensor Technology, vol. SPIE-2747, Orlando, FL, Apr. 8-12, 1996, pp. 48-60.

[12] D. R. Sheen, S. J. Shackman, N. L. Vandenberg, D. L. Wiseman, L. P. Elenbogen, and R. F. Rawson, "The P-3 ultra-wideband SAR: Description and examples," in Proc. IEEE Nat. Radar Conf., May 13-16, 1996, pp. $50-53$.

[13] B. T. Binder, M. F. Toups, S. Ayasli, and E. M. Adams, "SAR foliage penetration phenomenology of tropical rain forest and Northern U.S. Forest," in Rec. IEEE Int. Radar Conf., May 8-11, 1995, pp. 158-163.

[14] L. A. Bessette, S. M. Crooks, and S. Ayasli, "P-3 ultra-wideband SAR, Grayling, Michigan: Target and clutter phenomenology," in Proc. EEE Radar Conf., Apr. 20-22, 1999, pp. 125-129.

[15] M. I. Mirkin, C. F. Lee, T. O. Grosch, B. E. Hodges, S. Ayasli, K. Kappra, and K. Sturgess, "Results on ground penetration SAR phenomenology from June 1993 Yuma experiment," in Rec. IEEE Int. Radar Conf., May 8-11, 1995, pp. 164-170.

[16] J. K. Jao, C. F. Lee, and S. Ayasli, "Coherent spatial filtering for SAR detection of stationary targets," IEEE Trans. Aerosp. Electron. Syst., vol. 35, pp. 614-626, Apr. 1999.

[17] R. K. Raney, "Synthethc aperture imaging radar and moving targets," IEEE Trans. Aerosp. Electron. Syst., vol. AES-7, pp. 499-505, May 1971. 
[18] S. Werness, W. Carrara, L. Joyce, and D. Franczak, "Moving target imaging for SAR data," IEEE Trans. Aerosp. Electron. Syst., vol. 26, pp. 57-66, Jan. 1990.

[19] R. P. Perry, R. C. Dipietro, and R. L. Fante, "SAR imaging of moving targets," IEEE Trans. Aerosp. Electron. Syst., vol. 35, pp. 57-66, Jan. 1999.

[20] M. Kirscht, "Detection, velocity estimation and imaging of moving targets with single-channel SAR," in Proc. EUSAR'98, pp. 587-590.

[21] S. Barbarossa, "Detection and imaging of moving objects with synthetic aperture radar-Part 1: Optimal detection and parameter estimation theory," Proc. Inst. Elect. Eng. F, vol. 139, pp. 79-88, Feb. 1992.

[22] — "Detection and imaging of moving objects with synthetic aperture radar-Part I: Joint time-frequency analysis by Wigner-Ville distribution," Proc. Inst. Elect. Eng. F, vol. 139, pp. 89-97, Feb. 1992.

[23] S. Barbarossa and A. Farina, "Space-time-frequency processing of synthetic aperture radar signals," IEEE Trans. Aerosp. Electron. Syst., vol. 30, no. 2, pp. 341-358, Apr. 1994.

[24] H. Yang and M. Soumekh, "Blind-velocity SAR/ISAR imaging of a moving target in a stationary background," IEEE Trans. Image Processing, vol. 2, pp. 80-95, Jan. 1993.

[25] M. Soumekh, Fourier Array Imaging, Chapter 5. Englewood Cliffs, NJ: Prentice-Hall, 1994.

[26] R. Bamler, "A comparison of range-Doppler and wavenumber domain SAR focusing algorithms," IEEE Trans. Geosci. Remote Sensing, vol. 30, pp. 706-713, July 1992.
[27] C. Cafforio, C. Prati, and F. Rocca, "SAR data focusing using seismic migration techniques," IEEE Trans. Aerosp. Electron. Syst., vol. 27, pp. 194-206, Mar. 1991.

[28] R. H. Stolt, "Migration by Fourier transform," Geophysics, vol. 43, pp. 23-46, Feb. 1978.

[29] J. D. Murray, Asymptotic Analysis. New York: Springer-Verlag, 1984.

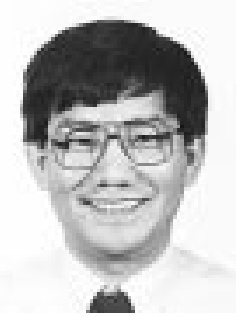

Jen King Jao (S'70-M'75) received the B.S. degree in electrophysics from National Chiao-Tung University, Hsinchu, Taiwan, R.O.C., in 1968, and the Ph.D. degree in electrical engineering from the Massachusetts Institute of Technology (MIT), Cambridge, in 1975.

Upon graduation, he joined the Riverside Research Institute and Raytheon Company. In 1979, he joined the MIT Lincoln Laboratory, Lexington, and since then has been active in air defense sensor phenomenology and technology development, particularly the area of airborne pulse-Doppler radar as well as radar clutter measurement and analysis. His current work concentrates on airborne radar ground surveillance investigations including the development of both single-aperture SAR and multichannel array radar technology for ground moving target imaging and detection. 